\title{
Palladium-Catalyzed Synthesis of 1-Alkylphosphonium Salts from 1-Alkenes
}

\author{
Mieko Arisawa and Masahiko Yamaguchi*广 \\ Department of Organic Chemistry, Graduate School of Pharmaceutical Sciences, \\ Tohoku University, Aoba, Sendai 980-8578, Japan \\ ${ }^{\dagger}$ Tohoku University 21 st Century COE Program CRESCENDO
}

\section{Supplementary Materials}

${ }^{1} \mathrm{H}-\mathrm{NMR},{ }^{13} \mathrm{C}-\mathrm{NMR}$, and ${ }^{31} \mathrm{P}-\mathrm{NMR}$ spectra were obtained on a Varian Mercury (400 MHz). Chemical shift values are given in ppm relative to internal $\mathrm{Me}_{4} \mathrm{Si}$. IR spectra were recorded on a JASCO FT/IR410. MS spectra were taken with a JEOL JMS-DX303 or a JEOL JMS-AX500 or 700. Elemental analyses were conducted with a Yanaco CHN corder MT-5. Melting Points were determined with a Yanaco micro melting point apparatus without correction.

Ethyltriphenylphosphonium Bis(trifluoromethanesulfonyl)imidate (Table 2, entry 1). In a two-necked flask equipped with a reflux condenser were placed triphenylphosphine ( $5 \mathrm{mmol}, 1.31 \mathrm{~g})$, $\mathrm{Pd}_{2} \mathrm{dba}_{3} \cdot \mathrm{CHCl}_{3}(0.1 \mathrm{~mol} \%, 5.2 \mathrm{mg})$, and bis(trifluoromethanesulfonyl)imide (5.5 mmol, $\left.1.55 \mathrm{~g}\right)$ in chlorobenzene $(6 \mathrm{~mL})$ under an argon atmosphere. After substituting the argon balloon to ethylene, the solution was heated at $65^{\circ} \mathrm{C}$ for $5 \mathrm{~h}$, and then the solution was concentrated. The residue was washed with ether/hexane, and recrystallized from ethanol giving the title compound $(2.79 \mathrm{~g}, 98 \%)$ as colorless solid. Mp 97.0-97.5 ${ }^{\circ} \mathrm{C}(\mathrm{EtOH}) .{ }^{1} \mathrm{H}-\mathrm{NMR}\left(400 \mathrm{MHz}, \mathrm{CDCl}_{3}\right) \delta 1.37$ (3H, dt, J = 19.6, 7.2 $\mathrm{Hz}), 3.21-3.28(2 \mathrm{H}, \mathrm{m}), 7.67(6 \mathrm{H}, \mathrm{dd}, \mathrm{J}=11.6,7.2 \mathrm{~Hz}), 7.71(6 \mathrm{H}, \mathrm{dt}, \mathrm{J}=7.2,3.6 \mathrm{~Hz}), 7.83(3 \mathrm{H}, \mathrm{t}, \mathrm{J}=$ $7.2 \mathrm{~Hz}) .{ }^{13} \mathrm{C}-\mathrm{NMR}\left(100 \mathrm{MHz}, \mathrm{CDCl}_{3}\right) \delta 6.4(\mathrm{~d}, \mathrm{~J}=4.5 \mathrm{~Hz}), 16.2(\mathrm{~d}, \mathrm{~J}=52.3 \mathrm{~Hz}), 117.5(\mathrm{~d}, \mathrm{~J}=85.7$ $\mathrm{Hz}), 119.8$ (q, J = 319.8 Hz), 130.5 (d, J = 12.1 Hz), 133.2 (d, J = 9.8 Hz), 135.2 (d, J = 3.0 Hz). ${ }^{31} \mathrm{P}-$ NMR (162 MHz, $\left.\mathrm{CDCl}_{3}\right) \delta$ 24.9. IR (KBr) 3062, 2966, 2938, 1441, 1348, 1200, 1060, $735 \mathrm{~cm}^{-1}$. MS (FAB) $\mathrm{m} / \mathrm{z} 291\left(\mathbf{M}^{+}-\mathrm{Tf}_{2} \mathrm{~N}\right)$. Anal. Calcd. for $\mathrm{C}_{22} \mathrm{H}_{20} \mathrm{~F}_{6} \mathrm{NO}_{4} \mathrm{PS}_{2}: \mathrm{C}, 46.24 \% \mathrm{H}, 3.53 \% \mathrm{~N}, 2.45 \% \mathrm{~S}$, $11.22 \%$. Found: C, $46.08 \% \mathrm{H}, 3.55 \% \mathrm{~N}, 2.45 \% \mathrm{~S}, 11.27 \%$.

1-Propyltriphenylphosphonium Bis(trifluoromethanesulfonyl)imidate (Table 2, entry 2). Colorless solid. Mp 76.5-77.5 ${ }^{\circ} \mathrm{C}(\mathrm{EtOH}) .{ }^{1} \mathrm{H}-\mathrm{NMR}\left(400 \mathrm{MHz}, \mathrm{CDCl}_{3}\right) \delta 1.17(3 \mathrm{H}, \mathrm{t}, \mathrm{J}=7.2 \mathrm{~Hz})$, $1.69(2 \mathrm{H}$, septet, J = 7.2 Hz), 3.11-3.18 (2H, m), $7.67(6 \mathrm{H}, \mathrm{dd}, \mathrm{J}=13.2,7.2 \mathrm{~Hz}), 7.71(6 \mathrm{H}, \mathrm{dt}, \mathrm{J}=7.2$, $3.6 \mathrm{~Hz}), 7.82(3 \mathrm{H}, \mathrm{dt}, \mathrm{J}=7.2,1.6 \mathrm{~Hz}) .{ }^{13} \mathrm{C}-\mathrm{NMR}\left(100 \mathrm{MHz}, \mathrm{CDCl}_{3}\right) \delta 15.0(\mathrm{~d}, \mathrm{~J}=17.4 \mathrm{~Hz}), 16.4(\mathrm{~d}$, $\mathrm{J}=4.5 \mathrm{~Hz}), 24.1(\mathrm{~d}, \mathrm{~J}=50.7 \mathrm{~Hz}), 117.8(\mathrm{~d}, \mathrm{~J}=86.4 \mathrm{~Hz}), 119.9(\mathrm{q}, \mathrm{J}=319.7 \mathrm{~Hz}), 130.6(\mathrm{~d}, \mathrm{~J}=12.9$ $\mathrm{Hz}), 133.2(\mathrm{~d}, \mathrm{~J}=9.8 \mathrm{~Hz}), 135.3(\mathrm{~d}, \mathrm{~J}=3.0 \mathrm{~Hz}) .{ }^{31} \mathrm{P}-\mathrm{NMR}\left(162 \mathrm{MHz}, \mathrm{CDCl}_{3}\right) \delta 22.7 . \mathrm{IR}(\mathrm{KBr})$ 3064, 2931, 1349, 1196, 1057, $739 \mathrm{~cm}^{-1}$. MS (FAB) m/z $305\left(\mathbf{M}^{+}-\mathrm{Tf}_{2} \mathrm{~N}\right)$. Anal. Calcd. for $\mathrm{C}_{23} \mathrm{H}_{22} \mathrm{~F}_{6} \mathrm{NO}_{4} \mathrm{PS}_{2}$ : C, $47.18 \% \mathrm{H}, 3.79 \% \mathrm{~N}, 2.39 \% \mathrm{~S}, 10.95 \%$. Found: C, $47.09 \% \mathrm{H}, 3.67 \% \mathrm{~N}, 2.39 \%$ $\mathrm{S}, 11.06 \%$.

1-Butyltriphenylphosphonium Bis(trifluoromethanesulfonyl)imidate (Table 2, entry 3). 20 equivalents of 1-butene were reacted. Colorless solid. Mp 83.5-84.0 ${ }^{\circ} \mathrm{C}(\mathrm{EtOH}) .{ }^{1} \mathrm{H}-\mathrm{NMR}(400$ 
$\left.\mathrm{MHz}, \mathrm{CDCl}_{3}\right) \delta 0.93(3 \mathrm{H}, \mathrm{t}, \mathrm{J}=7.6 \mathrm{~Hz}), 1.57-1.63(4 \mathrm{H}, \mathrm{m}), 3.14-3.23(2 \mathrm{H}, \mathrm{m}), 7.66(6 \mathrm{H}, \mathrm{dd}, \mathrm{J}=$ $12.4,8.0 \mathrm{~Hz}), 7.72(6 \mathrm{H}, \mathrm{dt}, \mathrm{J}=8.0,3.6 \mathrm{~Hz}), 7.83(3 \mathrm{H}, \mathrm{dt}, \mathrm{J}=8.0,2.4 \mathrm{~Hz}) .{ }^{13} \mathrm{C}-\mathrm{NMR}(100 \mathrm{MHz}$, $\left.\mathrm{CDCl}_{3}\right) \delta 13.4,22.2(\mathrm{~d}, \mathrm{~J}=51.5 \mathrm{~Hz}), 23.6(\mathrm{~d}, \mathrm{~J}=15.9 \mathrm{~Hz}), 24.4(\mathrm{~d}, \mathrm{~J}=4.6 \mathrm{~Hz}), 117.9(\mathrm{~d}, \mathrm{~J}=85.6$ $\mathrm{Hz}), 119.9$ (q, J = 319.0 Hz), 130.6 (d, J = 12.1 Hz), 133.3 (d, J = 9.9 Hz), 135.3 (d, J = 3.0 Hz). ${ }^{31} \mathrm{P}-$ NMR (162 MHz, $\mathrm{CDCl}_{3}$ ) $\delta$ 23.1. IR (KBr) 3064, 2969, 2937, 1441, 1354, 1201, $1049 \mathrm{~cm}^{-1}$. MS (FAB) $\mathrm{m} / \mathrm{z} 319\left(\mathrm{M}^{+}-\mathrm{Tf}_{2} \mathrm{~N}\right)$. Anal. Calcd. for $\mathrm{C}_{24} \mathrm{H}_{24} \mathrm{~F}_{6} \mathrm{NO}_{4} \mathrm{PS}_{2}: \mathrm{C}, 48.08 \% \mathrm{H}, 4.03 \% \mathrm{~N}, 2.34 \% \mathrm{~S}$, $10.70 \%$. Found: C, $47.92 \% \mathrm{H}, 3.78 \% \mathrm{~N}, 2.35 \% \mathrm{~S}, 10.73 \%$.

1-Pentyltriphenylphosphonium Bis(trifluoromethanesulfonyl)imidate (Table 2, entry 4). 20 equivalents of 1-pentene were reacted. Colorless solid. Mp 96.5-97.0 ${ }^{\circ} \mathrm{C}\left(\mathrm{EtOH}: \mathrm{Et}_{2} \mathrm{O}=2: 1\right) .{ }^{1} \mathrm{H}-$ NMR (400 MHz, $\left.\mathrm{CDCl}_{3}\right) \delta 0.84(3 \mathrm{H}, \mathrm{t}, \mathrm{J}=7.2 \mathrm{~Hz}), 1.31(2 \mathrm{H}$, sixtet, J = 7.6 Hz), $1.52(2 \mathrm{H}$, quintet, J $=7.2 \mathrm{~Hz}), 1.62(2 \mathrm{H}$, sixtet, J = 7.2 Hz), 3.12-3.19 (2H, m), $7.66(6 \mathrm{H}, \mathrm{dd}, \mathrm{J}=12.4,7.2 \mathrm{~Hz}), 7.72(6 \mathrm{H}$, $\mathrm{dt}, \mathrm{J}=7.2,3.6 \mathrm{~Hz}), 7.83(3 \mathrm{H}, \mathrm{dt}, \mathrm{J}=7.2,1.6 \mathrm{~Hz}) .{ }^{13} \mathrm{C}-\mathrm{NMR}\left(100 \mathrm{MHz}, \mathrm{CDCl}_{3}\right) \delta$ 13.5, 21.9, $22.1(\mathrm{~d}$, $\mathrm{J}=4.6 \mathrm{~Hz}), 22.3(\mathrm{~d}, \mathrm{~J}=50.8 \mathrm{~Hz}), 32.3(\mathrm{~d}, \mathrm{~J}=15.1 \mathrm{~Hz}), 117.9(\mathrm{~d}, \mathrm{~J}=85.7 \mathrm{~Hz}), 119.9$ (q, J = 318.2 $\mathrm{Hz}), 130.6$ (d, J = 12.9 Hz), 133.3 (d, J = 9.8 Hz), 135.3 (d, J = 3.0 Hz). ${ }^{31} \mathrm{P}-\mathrm{NMR}(162 \mathrm{MHz}$,

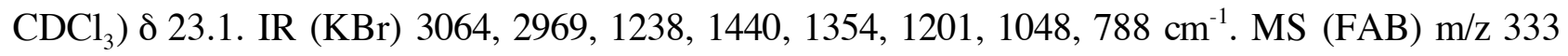
$\left(\mathbf{M}^{+}-\mathrm{Tf}_{2} \mathrm{~N}\right)$. Anal. Calcd. for $\mathrm{C}_{25} \mathrm{H}_{26} \mathrm{~F}_{6} \mathrm{NO}_{4} \mathrm{PS}_{2}: \mathrm{C}, 48.94 \% \mathrm{H}, 4.27 \% \mathrm{~N}, 2.28 \% \mathrm{~S}, 10.45 \%$. Found: C, $48.85 \% \mathrm{H}, 4.21 \% \mathrm{~N}, 2.27 \% \mathrm{~S}, 10.45 \%$.

1-Hexyltriphenylphosphonium Bis(trifluoromethanesulfonyl)imidate (Table 2, entry 5). 20 equivalents of 1-hexene were reacted. Colorless solid. $\mathrm{Mp} 90.0-90.5^{\circ} \mathrm{C}\left(\mathrm{EtOH}: \mathrm{Et}_{2} \mathrm{O}=2: 1\right) .{ }^{1} \mathrm{H}-$ NMR $\left(400 \mathrm{MHz}, \mathrm{CDCl}_{3}\right) \delta 0.84(3 \mathrm{H}, \mathrm{t}, \mathrm{J}=7.2 \mathrm{~Hz}), 1.24-1.28(4 \mathrm{H}, \mathrm{m}), 1.52-1.58(2 \mathrm{H}, \mathrm{m}), 1.59-1.64$ $(2 \mathrm{H}, \mathrm{m}), 3.12-3.19(2 \mathrm{H}, \mathrm{m}), 7.66(6 \mathrm{H}, \mathrm{dd}, \mathrm{J}=12.4,7.2 \mathrm{~Hz}), 7.72(6 \mathrm{H}, \mathrm{dt}, \mathrm{J}=7.2,3.6 \mathrm{~Hz}), 7.83(3 \mathrm{H}$, $\mathrm{dt}, \mathrm{J}=7.2,1.6 \mathrm{~Hz}) .{ }^{13} \mathrm{C}-\mathrm{NMR}\left(100 \mathrm{MHz}, \mathrm{CDCl}_{3}\right) \delta 13.8,22.2,22.3(\mathrm{~d}, \mathrm{~J}=52.3 \mathrm{~Hz}), 22.4(\mathrm{~d}, \mathrm{~J}=4.6$ Hz), 30.0 (d, J = 15.1 Hz), 31.0, 117.9 (d, J = 85.6 Hz), 119.9 (q, J = 319.7 Hz), 130.6 (d, J = 12.1 $\mathrm{Hz}), 133.3$ (d, J = 9.8 Hz), 135.3 (d, J = 3.0 Hz). ${ }^{31} \mathrm{P}-\mathrm{NMR}\left(162 \mathrm{MHz}, \mathrm{CDCl}_{3}\right) \delta$ 23.0. IR (KBr) 3065, 2935, 2872, 1352, 1195, $1058 \mathrm{~cm}^{-1}$. MS (FAB) m/z $347\left(\mathbf{M}^{+}-\mathrm{Tf}{ }_{2} \mathrm{~N}\right)$. Anal. Calcd. for $\mathrm{C}_{26} \mathrm{H}_{28} \mathrm{~F}_{6} \mathrm{NO}_{4} \mathrm{PS}_{2}: \mathrm{C}, 49.76 \% \mathrm{H}, 4.50 \% \mathrm{~N}, 2.23 \% \mathrm{~S}, 10.22 \%$. Found: C, 49.72\% H, 4.60\% N, $2.13 \%$ S, $10.33 \%$.

Ethyltris(p-chlorophenyl)phosphonium Bis(trifluoromethanesulfonyl)imidate (Table 2, entry 6). Colorless solid. $\mathrm{Mp} 84.5-85.5^{\circ} \mathrm{C}\left(\mathrm{EtOH}: \mathrm{Et}_{2} \mathrm{O}=2: 1\right) .{ }^{1} \mathrm{H}-\mathrm{NMR}\left(400 \mathrm{MHz}, \mathrm{CDCl}_{3}\right)$ $\delta 1.35(3 \mathrm{H}, \mathrm{dt}, \mathrm{J}=20.8,7.2 \mathrm{~Hz}), 3.25-3.37(2 \mathrm{H}, \mathrm{m}), 7.62(6 \mathrm{H}, \mathrm{dd}, \mathrm{J}=12.4,8.0 \mathrm{~Hz}), 7.70(6 \mathrm{H}, \mathrm{dd}, \mathrm{J}=$ 8.0, 2.4 Hz). ${ }^{13} \mathrm{C}-\mathrm{NMR}\left(100 \mathrm{MHz}, \mathrm{CDCl}_{3}\right) \delta 6.3,16.1(\mathrm{~d}, \mathrm{~J}=51.5 \mathrm{~Hz}), 115.3(\mathrm{~d}, \mathrm{~J}=88.6 \mathrm{~Hz}), 119.8$ $(\mathrm{q}, \mathrm{J}=319.8 \mathrm{~Hz}), 131.2(\mathrm{~d}, \mathrm{~J}=12.9 \mathrm{~Hz}), 134.6(\mathrm{~d}, \mathrm{~J}=11.4 \mathrm{~Hz}), 142.9 .{ }^{31} \mathrm{P}-\mathrm{NMR}(162 \mathrm{MHz}$,

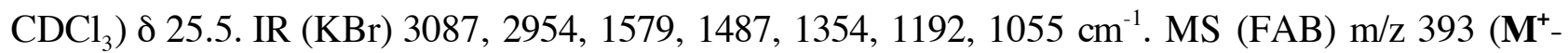
$\mathrm{Tf}_{2} \mathrm{~N}$ ). Anal. Calcd. for $\mathrm{C}_{22} \mathrm{H}_{17} \mathrm{Cl}_{3} \mathrm{~F}_{6} \mathrm{NO}_{4} \mathrm{PS}_{2}$ : C, 39.16\% H, 2.54\% N, 2.08\% Cl, $15.76 \% \mathrm{~S}, 9.50 \%$. Found: C, $39.15 \% \mathrm{H}, 2.54 \% \mathrm{~N}, 2.08 \% \mathrm{Cl}, 15.65 \% \mathrm{~S}, 9.35 \%$.

1-Propyltris (p-chlorophenyl)phosphonium Bis(trifluoromethanesulfonyl)imidate (Table 2, entry 7). Colorless solid. Mp $148.5-149.0{ }^{\circ} \mathrm{C}\left(\mathrm{EtOH}: \mathrm{Et}_{2} \mathrm{O}=2: 1\right) .{ }^{1} \mathrm{H}-\mathrm{NMR}\left(400 \mathrm{MHz}, \mathrm{CDCl}_{3}\right)$ $\delta 1.16(3 \mathrm{H}, \mathrm{t}, \mathrm{J}=7.2 \mathrm{~Hz}), 1.63(2 \mathrm{H}$, septet, $\mathrm{J}=7.2 \mathrm{~Hz}), 3.14-3.21(2 \mathrm{H}, \mathrm{m}), 7.62(6 \mathrm{H}, \mathrm{dd}, \mathrm{J}=12.4,8.0$ $\mathrm{Hz}), 7.69(6 \mathrm{H}, \mathrm{dd}, \mathrm{J}=8.0,3.2 \mathrm{~Hz}) .{ }^{13} \mathrm{C}-\mathrm{NMR}\left(100 \mathrm{MHz}, \mathrm{CDCl}_{3}\right) \delta 14.8(\mathrm{~d}, \mathrm{~J}=17.4 \mathrm{~Hz}), 16.3(\mathrm{~d}, \mathrm{~J}$ 
= 4.5 Hz), $23.7(\mathrm{~d}, \mathrm{~J}=50.0 \mathrm{~Hz}), 115.6(\mathrm{~d}, \mathrm{~J}=88.7 \mathrm{~Hz}), 119.8(\mathrm{q}, \mathrm{J}=319.7 \mathrm{~Hz}), 131.2(\mathrm{~d}, \mathrm{~J}=12.8$ $\mathrm{Hz}), 134.6(\mathrm{~d}, \mathrm{~J}=11.4 \mathrm{~Hz}), 142.9$ (d, J = 3.0 Hz). ${ }^{31} \mathrm{P}-\mathrm{NMR}\left(162 \mathrm{MHz}, \mathrm{CDCl}_{3}\right) \delta$ 23.1. IR $(\mathrm{KBr})$ 3091, 2974, 1579, 1486, 1351, 1201, 1055, 822, $760 \mathrm{~cm}^{-1}$. MS (FAB) m/z $407\left(\mathbf{M}^{+}-\mathrm{Tf}_{2} \mathrm{~N}\right)$. Anal. Calcd. for $\mathrm{C}_{23} \mathrm{H}_{19} \mathrm{Cl}_{3} \mathrm{~F}_{6} \mathrm{NO}_{4} \mathrm{PS}_{2}: \mathrm{C}, 40.10 \% \mathrm{H}, 2.78 \% \mathrm{~N}, 2.03 \% \mathrm{Cl}, 15.44 \% \mathrm{~S}, 9.31 \%$. Found: $\mathrm{C}$, $40.26 \% \mathrm{H}, 2.83 \% \mathrm{~N}, 1.89 \% \mathrm{Cl}, 15.26 \% \mathrm{~S}, 9.17 \%$.

\section{1-Butyltris (p-chlorophenyl)phosphonium Bis(trifluoromethanesulfonyl)imidate (Table 2,} entry 8). 20 equivalents of 1-butene were reacted. Colorless solid. Mp $144.0-145.0{ }^{\circ} \mathrm{C}(\mathrm{EtOH}$ : $\left.\mathrm{Et}_{2} \mathrm{O}=2: 1\right) .{ }^{1} \mathrm{H}-\mathrm{NMR}\left(400 \mathrm{MHz}, \mathrm{CDCl}_{3}\right) \delta 0.92(3 \mathrm{H}, \mathrm{t}, \mathrm{J}=7.2 \mathrm{~Hz}), 1.48-1.62$ (4H, m), 3.18-3.25 $(2 \mathrm{H}, \mathrm{m}), 7.62(6 \mathrm{H}, \mathrm{dd}, \mathrm{J}=12.4,8.0 \mathrm{~Hz}), 7.70(6 \mathrm{H}, \mathrm{dd}, \mathrm{J}=8.0,3.2 \mathrm{~Hz}) .{ }^{13} \mathrm{C}-\mathrm{NMR}\left(100 \mathrm{MHz}, \mathrm{CDCl}_{3}\right)$

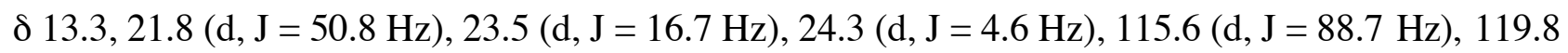
$(\mathrm{q}, \mathrm{J}=319.0 \mathrm{~Hz}), 131.2(\mathrm{~d}, \mathrm{~J}=13.6 \mathrm{~Hz}), 134.6(\mathrm{~d}, \mathrm{~J}=10.7 \mathrm{~Hz}), 143.0 .{ }^{31} \mathrm{P}-\mathrm{NMR}(162 \mathrm{MHz}$, $\mathrm{CDCl}_{3}$ ) $\delta$ 23.6. IR (KBr) 3092, 2966, 1579, 1352, 1199, 1056, 819, $759 \mathrm{~cm}^{-1} . \mathrm{MS}$ (FAB) m/z 421 $\left(\mathrm{M}^{+}-\mathrm{Tf}_{2} \mathrm{~N}\right)$. Anal. Calcd. for $\mathrm{C}_{24} \mathrm{H}_{21} \mathrm{Cl}_{3} \mathrm{~F}_{6} \mathrm{NO}_{4} \mathrm{PS}_{2}: \mathrm{C}, 41.01 \% \mathrm{H}, 3.01 \% \mathrm{~N}, 1.99 \% \mathrm{Cl}, 15.13 \% \mathrm{~S}$, 9.12\%. Found: C, $40.81 \% \mathrm{H}, 3.02 \% \mathrm{~N}, 1.97 \% \mathrm{Cl}, 14.95 \% \mathrm{~S}, 8.90 \%$.

1-Pentyltris( $p$-chlorophenyl)phosphonium Bis(trifluoromethanesulfonyl)imidate (Table 2 , entry 9). In a two-necked flask equipped with a reflux condenser were placed tris $(p$ chlorophenyl)phosphine (1 mmol, $365 \mathrm{mg}$ ), $\operatorname{Pd}_{2} \mathrm{dba}_{3} \cdot \mathrm{CHCl}_{3} \quad(1 \mathrm{~mol} \%, 10.4 \mathrm{mg}$ ), bis(trifluoromethanesulfonyl)imide $(1.1 \mathrm{mmol}, 310 \mathrm{mg})$, and 1-pentene $(5 \mathrm{mmol}, 0.55 \mathrm{~mL})$ in chlorobenzene $(3 \mathrm{~mL})$ under an argon atmosphere. The solution was heated at $65{ }^{\circ} \mathrm{C}$ for $24 \mathrm{~h}$, and concentrated. The residue was washed with ether/hexane, and recrystallized from ethanol : ether $=2$ : 1 giving the title compound (648.4 mg, 91\%) as colorless solid. $\mathrm{Mp} 85.5-86.0{ }^{\circ} \mathrm{C}\left(\mathrm{EtOH}^{2} \mathrm{Et}_{2} \mathrm{O}=2\right.$ : 1). ${ }^{1} \mathrm{H}-\mathrm{NMR}\left(400 \mathrm{MHz}, \mathrm{CDCl}_{3}\right) \delta 0.84(3 \mathrm{H}, \mathrm{t}, \mathrm{J}=7.2 \mathrm{~Hz}), 1.31(2 \mathrm{H}$, septet, $\mathrm{J}=7.6 \mathrm{~Hz}), 1.49-1.57$ $(4 \mathrm{H}, \mathrm{m}), 3.15-3.22(2 \mathrm{H}, \mathrm{m}), 7.61(6 \mathrm{H}, \mathrm{dd}, \mathrm{J}=12.4,8.0 \mathrm{~Hz}), 7.68(6 \mathrm{H}, \mathrm{dd}, \mathrm{J}=8.0,2.0 \mathrm{~Hz}) .{ }^{13} \mathrm{C}-\mathrm{NMR}$ $\left(100 \mathrm{MHz}, \mathrm{CDCl}_{3}\right) \delta$ 13.7, 22.05, $22.06(\mathrm{~d}, \mathrm{~J}=50.0 \mathrm{~Hz}), 22.2(\mathrm{~d}, \mathrm{~J}=4.5 \mathrm{~Hz}), 32.3(\mathrm{~d}, \mathrm{~J}=16.7 \mathrm{~Hz})$, 115.5 (d, J = 87.9 Hz), 119.7 (q, J = 318.2 Hz), $131.1(\mathrm{~d}, \mathrm{~J}=12.8 \mathrm{~Hz}), 134.4$ (d, J = 11.4 Hz), 142.8 $(\mathrm{d}, \mathrm{J}=3.8 \mathrm{~Hz}) .{ }^{31} \mathrm{P}-\mathrm{NMR}\left(162 \mathrm{MHz}, \mathrm{CDCl}_{3}\right) \delta$ 23.4. IR (KBr) 3090, 2957, 2872, 1580, 1353, 1201, 1065, 1012, 817, $760 \mathrm{~cm}^{-1}$. MS (FAB) m/z $435\left(\mathbf{M}^{+}-\mathrm{Tf}_{2} \mathrm{~N}\right)$. Anal. Calcd. for $\mathrm{C}_{25} \mathrm{H}_{23} \mathrm{Cl}_{3} \mathrm{~F}_{6} \mathrm{NO}_{4} \mathrm{PS}_{2}: \mathrm{C}$, $41.88 \% \mathrm{H}, 3.23 \% \mathrm{~N}, 1.95 \% \mathrm{Cl}, 14.84 \% \mathrm{~S}, 8.95 \%$. Found: C, $41.72 \% \mathrm{H}, 3.30 \% \mathrm{~N}, 1.92 \% \mathrm{Cl}$, $14.78 \% \mathrm{~S}, 8.80 \%$.

\section{1-Hexyltris(p-chlorophenyl)phosphonium Bis(trifluoromethanesulfonyl)imidate (Table 2,} entry 10). 5 equivalents of 1 -hexene were reacted. Colorless solid. $\mathrm{Mp} 80.0-81.0{ }^{\circ} \mathrm{C}(\mathrm{EtOH}) .{ }^{1} \mathrm{H}-$ NMR $\left(400 \mathrm{MHz}, \mathrm{CDCl}_{3}\right) \delta 0.85(3 \mathrm{H}, \mathrm{t}, \mathrm{J}=7.2 \mathrm{~Hz}), 1.23-1.28(4 \mathrm{H}, \mathrm{m}), 1.53-1.57(4 \mathrm{H}, \mathrm{m}), 3.20-3.28$ $(2 \mathrm{H}, \mathrm{m}), 7.61(6 \mathrm{H}, \mathrm{dd}, \mathrm{J}=12.4,8.0 \mathrm{~Hz}), 7.71(6 \mathrm{H}, \mathrm{dd}, \mathrm{J}=8.0,2.4 \mathrm{~Hz}) .{ }^{13} \mathrm{C}-\mathrm{NMR}\left(100 \mathrm{MHz}, \mathrm{CDCl}_{3}\right)$ ठ 13.7, $22.0(\mathrm{~d}, \mathrm{~J}=51.3 \mathrm{~Hz}), 22.2,22.3(\mathrm{~d}, \mathrm{~J}=4.5 \mathrm{~Hz}), 29.9$ (d, J = 16.7 Hz), 30.9, 115.6 (d, J = 87.9 Hz), 119.8 (q, J = 319.0 Hz), 131.2 (d, J = 12.9 Hz), 134.6 (d, J = 10.6 Hz), 142.9 (d, J = 3.8 Hz).

${ }^{31}$ P-NMR (162 MHz, $\mathrm{CDCl}_{3}$ ) $\delta$ 23.7. IR (KBr) 3090, 2957, 2871, 1580, 1353, 1202, 1065, $760 \mathrm{~cm}^{-1}$. MS (FAB) m/z $449\left(\mathbf{M}^{+}-\mathrm{Tf}_{2} \mathrm{~N}\right)$. Anal. Calcd. for $\mathrm{C}_{26} \mathrm{H}_{25} \mathrm{Cl}_{3} \mathrm{~F}_{6} \mathrm{NO}_{4} \mathrm{PS}_{2}: \mathrm{C}, 42.72 \% \mathrm{H}, 3.45 \% \mathrm{~N}$, $1.92 \% \mathrm{Cl}, 14.55 \% \mathrm{~S}, 8.77 \%$. Found: C, $42.76 \% \mathrm{H}, 3.37 \% \mathrm{~N}, 1.84 \% \mathrm{Cl}, 14.53 \% \mathrm{~S}, 8.51 \%$.

Reaction of $(\boldsymbol{E})$-2-hexene. In a two-necked flask equipped with a reflux condenser were placed tris(p-chlorophenyl)phosphine (1 mmol, $365 \mathrm{mg}), \mathrm{Pd}_{2} \mathrm{dba}_{3} \cdot \mathrm{CHCl}_{3}(1 \mathrm{~mol} \%, 10.4 \mathrm{mg})$, 
bis(trifluoromethanesulfonyl)imide $(1.1 \mathrm{mmol}, 310 \mathrm{mg})$, and $(E)$-2-hexene $(5 \mathrm{mmol}, 0.625 \mathrm{~mL})$ in chlorobenzene $(3 \mathrm{~mL})$ under an argon atmosphere. The solution was heated at $65{ }^{\circ} \mathrm{C}$ for $24 \mathrm{~h}$, and then was concentrated. The residue was washed with ether/hexane. ${ }^{1} \mathrm{H}-\mathrm{NMR}$ analysis using 1,1,2trichloroethane as internal standard indicated formation of hexyltris( $p$-chlorophenyl)phosphonium bis(trifluoromethanesulfonyl)imidate in $91 \%$ yield. The reaction of $(E)$-3-hexene proceeded analogously. 